\title{
FLAT BUNDLES WITH SOLVABLE HOLONOMY
}

\author{
WILLIAM M. GOLDMAN AND MORRIS W. HIRSCH
}

\begin{abstract}
Let $G$ be a solvable linear Lie group. We show that for every flat principal $G$-bundle $\xi$ over a $C W$-complex $M$, there is a finite-sheeted covering space $p: \hat{M} \rightarrow M$ such that $p^{*} \xi$ is trivial as a principal $G$-bundle. This result is used to show that every affine manifold with solvable fundamental group has a finite covering which is parallelizable.
\end{abstract}

In this note $M$ denotes a connected manifold or $\mathrm{CW}$-complex with fundamental group $\pi$ and $G$ denotes a Lie group. A bundle $\xi$ over $M$ with structure group $G$ is virtually trivial if and only if there is a finite covering space $p: \hat{M} \rightarrow M$ such that $p^{*} \xi$ is a trivial bundle. We call $\xi$ a flat $G$-bundle if the structure group has been reduced to a totally disconnected subgroup $\Gamma \subset G$, the holonomy group of the flat bundle. A vector bundle is flat if the associated principal bundle is flat.

The following result is known to others (e.g. D. Sullivan [6]) but there seems to be no published proof. Our purpose is to supply one.

THEOREM 1. Let $\xi$ be a flat vector bundle over $M$ whose holonomy group is finitely generated and contains a solvable subgroup of finite index. Then $\xi$ is virtually trivial.

The proof is based on

Theorem 2. Suppose $G$ is a solvable Lie group with finitely many components, which admits a faithful matrix representation. Let $\xi$ be a flat principal $G$-bundle over $M$ whose holonomy group is finitely generated. Then $\xi$ is virtually trivial.

Proof of Theorem 1 from Theorem 2. Let $\xi$ be a flat vector bundle over $M$, induced by a representation $\phi: \pi \rightarrow \mathrm{GL}(n ; R)$. Assuming the hypotheses of Theorem 1 , we may pass to a finite covering of $M$ and assume that $\phi(\pi)$ is actually solvable. Note that a subgroup of finite index in a finitely generated group is finitely generated.

Let $G$ denote the Zariski closure of $\phi(\pi)$ in $\operatorname{GL}(n ; R)$. Since any algebraic group has finitely many components and the Zariski closure of a solvable group is solvable, $G$ satisfies the hypotheses of Theorem 2 . Therefore the principal $G$-bundle induced by $\phi: \pi \rightarrow G$ is virtually trivial. Q.E.D.

We briefly recall how a homomorphism $h: \pi \rightarrow G$ induces a flat $G$-bundle, which we denote by $h_{\mathrm{b}}$. Let $p: \tilde{M} \rightarrow M$ denote a universal covering and identify $\pi$ as the group of deck transformations. Give $\pi$ the diagonal action on $\tilde{M} \times G$ defined by

Received by the editors June 4, 1980 and, in revised form, September 24, 1980.

1980 Mathematics Subject Classification. Primary 55R15, 57R22, 22E25; Secondary 57R15, 58 H99.

Key words and phrases. Solvable Lie group, flat bundle, covering space, affine manifold. 
$\gamma(\tilde{x}, g)=(\gamma \tilde{x}, h(\gamma) g)$. The orbit space $(\tilde{M} \times G) / \pi$ is the total space of a flat $G$-bundle over $M$; the projection sends the orbit of $(\tilde{x}, g)$ to $p(\tilde{x})$. The holonomy group is $h(\pi)$.

The following well-known results will be used. For convenience we indicate proofs.

LEMMA 3. Let $h_{t}: \pi \rightarrow G, 0<t<1$, be a continuous family of homomorphisms. Then $\left(h_{0}\right)_{b}$ and $\left(h_{1}\right)_{b}$ are isomorphic G-bundles. In particular, $\left(h_{1}\right)_{b}$ is a trivial $G$-bundle if $h_{0}$ is the trivial homomorphism.

Proof. There is a $G$-bundle $\xi$ over $M \times[0,1]$ such that $\left.\xi\right|_{M \times\{t\}}$ is isomorphic to $\left(h_{t}\right)_{b}$. Apply the covering homotopy theorem. Q.E.D.

LEMMA 4. Let $\phi: G \rightarrow H$ be a homomorphism which is a homotopy equivalence of Lie groups. Then $h_{\mathrm{b}}$ is a trivial G-bundle provided $(\phi \circ h)_{\mathrm{b}}$ is a trivial $\boldsymbol{H}$-bundle.

Proof. $\phi$ induces a homotopy equivalence of classifying spaces $B \phi: B G \rightarrow B H$. (In fact let $E G \rightarrow B G$ be the universal principal $G$-bundle. Then $H$ acts freely on the contractible space $E H=(E G \times H) / G$, where $G$ acts on $H$ via $\phi$ and $G$ acts diagonally on $E G \times H$. Therefore $E H \rightarrow(E H) / H$ is a universal bundle for $H$; but $B H=(E H) / H$ is naturally identified with $(E G) / G=B G$. Thus we can take $B H=B G$.)

Let $f: M \rightarrow B G$ be the classifying map for $h_{b}$ (as a $G$-bundle). Then $(B \phi) \circ f$ : $M \rightarrow B H$ classifies $(\phi \circ h)_{b}$. Since $(\phi \circ h)_{b}$ is trivial, $(B \phi) \circ f$ is null homotopic, and therefore, so is $f$. Q.E.D.

Proof of Theorem 2. Let $\xi$ be induced by $h: \pi \rightarrow G$ with $h(\pi)=\Gamma$. Let $G_{0} \subset G$ be the identity component. Then $h^{-1} G_{0}$ has finite index in $\pi$. We may replace $\xi$ by the induced bundle $\xi_{0}$ over the covering space corresponding to $h^{-1} G_{0}$. The holonomy group of $\xi_{0}$ is $\Gamma \cap G_{0} \subset G_{0}$. Therefore we may assume $G$ is connected.

The assumptions on $G$ imply that the commutator subgroup $G^{\prime}$ is closed and contractible (see Hochschild [3, Chapter XVIII, Theorem 3.2]). Therefore the natural homomorphism $\phi: G \rightarrow G / G^{\prime}$ is a homotopy equivalence. It follows from Lemma 4 that $\xi$ is virtually trivial provided $(\phi \circ h)_{b}$ is virtually trivial. Therefore we may replace $G$ by $G / G^{\prime}$. Hence we assume that $G$ is a connected abelian Lie group.

Now $\Gamma$ is a finitely generated abelian group, so there is a free abelian subgroup $\Gamma_{0} \subset \Gamma$ of finite index. Passing to the covering of $M$ corresponding to $h^{-1} \Gamma_{0}$ we assume $\Gamma$ is free abelian.

Let $\psi: \tilde{G} \rightarrow G$ be the universal covering group. Since $\Gamma$ is free abelian and $\tilde{G}$ is abelian, there is a homomorphism $\theta: \Gamma \rightarrow \tilde{G}$ with $\psi \circ \theta=$ identity of $\Gamma$.

Consider the commutative diagram

$$
\begin{array}{lll}
\boldsymbol{\theta} \theta \circ h & & \tilde{G} \\
& \rightarrow & \\
& & G
\end{array}
$$


Now $\tilde{G}$ is a 1-connected abelian Lie group; hence it is a vector group. For any $t \in R$ there is a homomorphism $u_{t}: \pi \rightarrow \tilde{G}, \gamma \mapsto t[(\theta \circ h)(\gamma)]$. Therefore $\psi \circ u_{t}$ : $\pi \rightarrow G, 0<t<1$, is a continuous family of homomorphisms connecting the trivial homomorphism to $h$. It follows from Lemma 3 that $h_{\mathrm{b}}$ is trivial. Q.E.D.

One particular case of interest occurs when $M$ is an affine manifold, i.e. when $M$ is locally modelled on an affine space so that overlapping charts are identified by affine maps. In that case the tangent bundle of $M$ is a flat vector bundle. From Theorem 1 we deduce the following:

Corollary 5. Let $M$ be an affine manifold whose fundamental group is finitely generated and has a solvable subgroup of finite index. Then $M$ is virtually parallelizable, i.e. $M$ has a finite covering which is parallelizable.

It is an open question whether every affine manifold is virtually parallelizable. In particular this would imply the famous conjecture (posed by Chern) that the Euler characteristic of a compact affine manifold must vanish. This was proved in the complete case by Kostant and Sullivan [8]. Using examples of Milnor [4], Smillie has constructed examples [5] of compact manifolds having nonzero Euler characteristic, whose tangent bundles are isomorphic to flat vector bundles. However it seems quite difficult to determine whether the manifolds can be made affine.

Auslander and Szczarba [7] give an example of a 5-dimensional compact flat orientable Riemannian manifold with solvable fundamental group, whose StiefelWhitney class $w_{2}$ is nonzero. This shows that not all orientable affine manifolds are stably parallelizable.

In [2] Hirsch and Thurston prove the following related result: Let $p: E \rightarrow M$ be a bundle with compact fiber $X$ and structure group $\Gamma \subset \operatorname{Homeo}(X)$. Suppose that $p$ is induced by a representation $\phi: \pi_{1}(M) \rightarrow \Gamma$ and $\Gamma$ is amenable (e.g. a finite extension of a solvable group). Then the induced homomorphism $p^{*}: H^{*}(M ; R) \rightarrow$ $H^{*}(E ; R)$ is injective. This is in some sense a cohomological version of Theorem 2 when the structure group is not a Lie group $G$ as above.

Using considerably deeper methods than these used here, Deligne and Sullivan [1] prove the sharp result that if $\xi$ is a flat complex vector bundle (i.e. a $C^{n}$-bundle induced by a representation $\left.\phi: \pi_{1}(M) \rightarrow \mathrm{GL}(n ; C)\right)$ over a compact polyhedron $M$, then $\xi$ is virtually trivial. As noted above there are flat real vector bundles (see [4]) which have nonzero Euler class and hence are nontrivial over any finite covering.

\section{REFERENCES}

1. P. Deligne and D. Sullivan, Fibrés vectoriels à groupe structural discret, C. R. Acad. Sci. Paris 281 (1975), 1081-1083.

2. M. Hirsch and W. Thurston, Foliated bundles, invariant measures and flat manifolds, Ann. of Math. (2) 101 (1975), 369-390.

3. G. Hochschild, The structure of Lie groups, Holden-Day, San Francisco, Calif., 1965.

4. J. Milnor, On the existence of a connection with curvature zero, Comment Math. Helv. 32 (1958), 215-223.

5. J. Smillie, Flat manifolds with nonzero Euler characteristic, Comment Math. Helv. 52 (1977), 453-456.

6. D. Sullivan, Conversation with W. Goldman, February, 1980. 
7. L Auslander and R. H. Szczarba, Characteristic classes of compact solomanifolds, Ann. of Math. (2) 76 (1962), 1-8.

8. B. Kostant and D. Sullivan, The Euler characteristic of an affine space form is zero, Bull. Amer. Math. Soc. 81 (1975), 937-938.

Department of Mathematics, University of California, Berkeley, California 94720 (Curtent address of M. W. Hirsch)

Current address (W. M. Goldman): Department of Mathematics, Massachusetts Institute of Technology, Cambridge, Massachusetts 02139 